\title{
Influence of diazonium and surfactant modification of the mesoporous material on its adsorption properties
}

\author{
Mariusz Sandomierski $^{1}$ (D) Kornelia Poniedziałek ${ }^{1} \cdot$ Katarzyna Bielicka-Daszkiewicz $^{1} \cdot$ Adam Voelkel $^{1}$
}

Received: 15 March 2019 / Accepted: 5 September 2019 / Published online: 13 September 2019

(c) The Author(s) 2019

\begin{abstract}
We describe a new and direct route to obtain Al-SBA-15-modified materials with different groups on their surface using diazonium salts. For comparison, modification using surfactants was also used. In the present study, we decided to examine the applicability of these materials in the adsorption of compounds released from dental fillings. The effectiveness of modification was proved by results of Fourier transform infrared spectroscopy and energy-dispersive spectrometry. The results indicate the formation of characteristic groups on the surface of the materials. The materials after modification are characterized by smaller surface area and pore volume, which is result of covering the surface with an aryl or surfactant layer. The adsorption properties change after modification and depend on the used modifier. Diazonium modification has a positive effect on sorptive properties when hydroxyl and dimethylamine groups are obtained. The methods of material modification presented in this work may in the future lead to the improvement in the properties of sorbents used in solid-phase extraction.
\end{abstract}

Keywords Adsorption · Mesoporous · Diazonium modification · Solid-phase extraction

\section{Introduction}

Ordered mesoporous materials were first reported in the 1990s. These materials are characterized by high surface area, pore volume and the ordering of pores with diameter in the mesopore range (2.0-10.0 nm) (Gomez-Cazalilla et al. 2007; Zeng et al. 2005). This group includes the material named Al-SBA-15 (Wang and Liu 2008). This material is a mesoporous silica (SBA15) with incorporated aluminum heteroatom in its structure and is synthesized under acidic conditions. Al-SBA-15 has a large pore size, thick walls and high thermal and hydrothermal stability. It is used in many fields such as, adsorption, catalysis, separation, nanoscience and as solid template for other materials (Kumaran et al. 2008; Szczodrowski et al. 2009). Despite many works describing methods of synthesis and modification of mesoporous

Electronic supplementary material The online version of this article (https://doi.org/10.1007/s11696-019-00926-3) contains supplementary material, which is available to authorized users.

Mariusz Sandomierski

mariuszsandomierski@wp.pl

1 Institute of Chemical Technology and Engineering, Poznan University of Technology, ul. Berdychowo 4, 60-965 Poznań, Poland aluminosilicates, there are a lot of various possibilities to improve its properties which have not been used so far.

One of the methods for the modification of aluminosilicates is the use of surfactants. The properties of the modified materials depend on the length of the chain in the surfactant molecule. Depending on the length of the carbon chain, during the modification process, a monolayer or bilayer is formed on the surface. It has been proven that the adsorption degree of organic compounds increased with the increase in the chain length of the surfactant used in the modification for hydrophilic compounds (Xie et al. 2012, 2013). In the case of hydrophobic compounds, this effect was not so visible but still they were adsorbed more by surfactant-modified zeolite compared to unmodified zeolite (Li et al. 2000).

Another interesting way to modify aluminosilicates is modification using diazonium salts. These salts have been previously used in the modification of many types of surfaces, but the modification and characterization of aluminosilicates have not been widely described (Mahouche-Chergui et al. 2011; Sandomierski et al. 2016, 2018a, b). Modification of aluminosilicates using surfactant allows only for the introduction of the aliphatic chains to the surface of the material. The modification using diazonium salts produces the organic layer with various substituents. The currently used materials modified with diazonium salts in the 
extraction processes mainly refers to the analysis of metal ions. Cui et al. (2012) prepared phenyl-iminodiacetic acidgrafted multiwalled carbon nanotubes for solid-phase extraction of iron, copper and lead ions from aqueous medium. It was found that the diazonium-modified sorbents have much higher adsorption capacity (Sitko et al. 2012). The surface modified using 4-sulfobenzendiazonium salt was used in ion chromatography. The obtained layer allowed to bind latex nanoparticles bearing quaternary triethylamine functional groups to carbon surface (Wahab et al. 2011). Diazonium salts were also used in reversible trapping of functional molecules process which indicates the potential of their use in extraction processes (Heimburger et al. 2016).

One of the important problems is the release of the components of modern dental fillings-resin-based composites (McCabe 1998). With the development of the new resin-based dental materials, problems with compounds released from restorative dental fillings in human teeth also occurred and these compounds affect human health. The leachable compounds may be released due to insufficient hardening of the dental filling (depends on the time of polymerization by light source exposure and its intensity), depth of cure and also the composition of dental filling which leads to incomplete conversion of monomer to polymer (Michelsen et al. 2012; Davidson and Feilzer 1997; Ruyter and Oysaed 1982; Gioka et al. 2005; Swift et al. 1995). The penetration of saliva, water, ethanol and other solvents in human mouth can elute the unreacted ingredients of dental filling (Rogalewicz et al. 2006; Manojlovic et al. 2011). Determination of these compounds is necessary because of the harmful effects such as, allergy from the released ingredients, cytotoxicity, toxicity and estrogenic activity (Koppula et al. 1995; Geurtsen et al. 1998; Wilder et al. 2000; Hashimoto and Nakamura 2000). The organic compounds such as nonreacted monomers and other components or degradation products of the fillings components leach from dental filling placed in a human tooth. Bisphenol-A diglycidyl methacrylate (Bis-GMA), 2-hydroxyethyl methacrylate (HEMA), urethane dimethacrylate (UDMA), triethylene glycol dimethacrylate (TEGDMA) and others were found as the released compounds.

It is important to propose the procedure of controlling the amount of leachables from dental fillings. Therefore in the present study, we decided to examine the applicability of new materials as adsorbents of compounds released from dental fillings. We report a new and direct route to obtain Al-SBA-15-modified materials with different groups on their surface using diazonium salts. Mesoporous aluminosilicates have not been previously modified using diazonium salts. For comparison, modification using surfactants was also used. In the study, we decided to examine the applicability of these materials in adsorption of compounds released from dental fillings which has not been described in literature so far. The methods of material modification presented in this work may in the future lead to the improvement of the properties of sorbents used in solid-phase extraction.

\section{Experimental}

\section{Materials}

\section{Chemicals}

The substrates for SBA materials synthesis and modification were purchased from Sigma-Aldrich: tetraethylorthosilicate (98\%), aluminium isopropoxide (98\%), PluronicF127, myristyltrimethylammonium bromide (MTAB, 99\%), cetyltrimethylammonium bromide (CTAB, 99\%), sodium nitrite (98\%), 4-aminobenzyl alcohol (98\%), 4-(Dimethylamino) aniline (97\%), sulfanilic acid (99\%), 4-toluidine (99\%) and $\mathrm{HCl}(36 \%)$. The chemical standards of elutable dental compounds were purchased from Sigma-Aldrich: camphor (96\%), dimethyl succinate (DBE-4, 98\%), dimethyl glutarate (DBE-5, 99\%), dimethyl adipate (DA, $\geq 99 \%$ ), camphor quinone $(\mathrm{CQ}, 97 \%)$, phenol, 2-hydroxyethyl methacrylate (HEMA, 97\%), 4-methoxyphenol (4-MP, $\geq 98.0 \%$ ), ethyl 4-(N,N-dimethyloamino) benzoate (EDMAB, $\geq 99 \%$ ), triethylene glycol dimethacrylate (TEGDMA, 95\%) and methanol was obtained from $\mathrm{POCH}$. Such elutables were mentioned in literature (Rogalewicz et al. 2006). The chemicals purchased from Sigma-Aldrich for artificial saliva preparation were potassium chloride ( $\geq 99 \%$ ), POCH: sodium chloride $(\geq 99.5 \%)$ and from ChemPur, potassium thiocyanate ( $\geq 99 \%$ ), potassium dihydrogen phosphate ( $\geq 99 \%$ ), sodium sulfate ( $\geq 99)$, ammonium chloride ( $\geq 99.5 \%$ ), calcium chloride dehydrate $(\geq 99.5 \%)$, sodium bicarbonate $(\geq 99.8 \%)$ and urea $(\geq 99.5 \%)$.

\section{Synthesis of AI-SBA-15 material}

SBA-15 materials were prepared according to the procedure reported by Grieken et al. (2009). Tetraethylorthosilicate $(13 \mathrm{~g})$ and aluminiumisopropoxide $(1.33 \mathrm{~g})$ were added to $15 \mathrm{ml}$ of aqueous $\mathrm{HCl}$ with $\mathrm{pH}$ equal to 1.5 . This mixture was stirred for $4 \mathrm{~h}$ at $25^{\circ} \mathrm{C}$ and then added to a second solution containing $6 \mathrm{~g}$ of Pluronic F127 in $225 \mathrm{ml}$ of aqueous $\mathrm{HCl}$ at $\mathrm{pH}$ 1.5. Afterwards, the solution was stirred for $20 \mathrm{~h}$ at $40{ }^{\circ} \mathrm{C}$ and aged at $110{ }^{\circ} \mathrm{C}$ for $24 \mathrm{~h}$ in an Autoclave Controller AM 1000 (Syl \& ANT) under static conditions. The solid product was recovered by filtration over a Büchner funnel, dried at $25^{\circ} \mathrm{C}$ overnight and calcined at $500{ }^{\circ} \mathrm{C}$ for $5 \mathrm{~h}$ using a heating rate of $0.4{ }^{\circ} \mathrm{C} \mathrm{min}{ }^{-1}$ in a Nabertherm $\mathrm{P}$ 330 furnace. 


\section{Surfactant modification of AI-SBA-15}

The sample was modified with myristyl- and cetyl-trimethyl ammonium bromides according to the method described by Xie et al. (2013). For this purpose, $500 \mathrm{ml}$ of the solution of the surfactant (myristyl- or cetyl-trimethyl ammonium bromide) with a concentration of $50 \mathrm{mmol} \mathrm{L}^{-1}$ was vigorously mixed with $10 \mathrm{~g}$ of Al-SBA- 15 at $50^{\circ} \mathrm{C}$ for $4 \mathrm{~h}$. After cooling to $25^{\circ} \mathrm{C}$, the product was centrifuged, washed with distilled water and methanol and then dried in the oven at $65^{\circ} \mathrm{C}$.

Methanol samples after washing were tested by GC analysis which showed no release of the surfactants.

\section{Diazonium modification of the AI-SBA-15}

Modification of sorbent was performed with in situ-generated diazonium salt according to the method presented in Sandomierski et al. (2018a). Amine was dissolved in distilled water and then cooled to a temperature below $5{ }^{\circ} \mathrm{C}$ in the ice bath. All reagents were mixed with a magnetic stirrer in a round-bottom flask, and then hydrochloric acid (36\%) was added to the mixture. Next, sodium nitrite dissolved in cold distilled water was added dropwise. The mesoporous sorbent was added to the flask after $30 \mathrm{~min}$ and stirred for $2 \mathrm{~h}$ at $25^{\circ} \mathrm{C}$. The modified material was thoroughly washed over a Büchner funnel using distilled water and methanol until it was clear and then dried in the oven at $65^{\circ} \mathrm{C}$. The following salts were used:

- 4-hydroxymethylbenzenediazonium salt $\left(-\mathrm{CH}_{2} \mathrm{OH}\right)$;

- 4-(N,N-dimethyl)benzenediazonium salt $\left(-\mathrm{N}\left(\mathrm{CH}_{3}\right)_{2}\right)$;

- 4-sulfobenzendiazonium salt $\left(-\mathrm{SO}_{3} \mathrm{H}\right)$;

- 4-methylbenzenediazonium salt $\left(-\mathrm{CH}_{3}\right)$.

Methanol samples after washing were examined by GC which showed no release of the diazonium salts.

\section{Techniques}

\section{Fourier transform infrared spectroscopy (FTIR)}

FTIR analysis was carried out using Vertex70 spectrometer, Bruker Optics. All materials were studied using single reflection, diamond ATR crystal. The tests were performed at a resolution of $0.5 \mathrm{~cm}^{-1}$ in the wavenumber range $4000-600 \mathrm{~cm}^{-1}$.

\section{Energy-dispersive spectrometry (EDS)}

EDS results were obtained using FEI Quanta FEG with field emission electron source under high vacuum mode, with accelerating voltage at $18 \mathrm{kV}$ equipped with EDS analyzer made by EDAX.

\section{Nitrogen adsorption/desorption measurement}

BET pore parameters, specific surface area and isotherm types were determined by the nitrogen adsorption isotherm technique by means of ASAP 2420 Micromeritrics analyser. Before the experiments, the samples were outgassed at $200{ }^{\circ} \mathrm{C}$ in a vacuum chamber.

\section{Inverse gas chromatography}

The surface properties of the materials were characterized by means of inverse gas chromatography (IGC). The measurements were performed using Surface Energy Analyzer produced by SMS Ltd., London. The materials under test were placed into the chromatographic columns (silanized glass columns, I.D. $4 \mathrm{~mm}$, length $30 \mathrm{~cm}$ ) by the tap-and-fill method. The measurements were carried out at $30{ }^{\circ} \mathrm{C}$. The temperature of injector as well as FID detector was $180{ }^{\circ} \mathrm{C}$. Helium was a carrier gas at a flow rate of $15 \mathrm{ml} / \mathrm{min}$.

Dispersive component of the free surface energy, $\gamma_{s}^{d}$, that expresses dispersive properties of the surface, was estimated according to Schultz-Lavielle procedure using n-alkanes as test compounds (hexane, heptane, octane, nonane, decane) (Schultz et al. 1987; Voelkel 2012). $\gamma_{s}^{d}$ parameter is calculated using the following equation:

$R \cdot T \cdot \ln V_{N}=2 \cdot N \cdot a \cdot \sqrt{\gamma_{s}^{d} \cdot \gamma_{l}^{d}}+C$

where $R$ is the gas constant, $8.314 \mathrm{~J} \cdot \mathrm{mol}^{-1} \mathrm{~K}^{-1} ; T$ is the temperature of measurement $(\mathrm{K}) ; V_{N}$ is the net retention volume $\left(\mathrm{m}^{3}\right) ; N$ is Avogadro's constant, $6.023 \cdot 10^{23} \mathrm{~mol}^{-1} ; a$ is the cross-sectional area of the adsorbate $\left(\mathrm{m}^{2}\right) ; \gamma_{s}^{d}$ is the dispersive component of surface-free energy $\left(\mathrm{mJ} / \mathrm{m}^{2}\right) ; \gamma_{l}^{d}$ is the dispersive component of the surface tension of the probe molecule in liquid state $\left(\mathrm{mJ} / \mathrm{m}^{2}\right)$; and $C$ is a constant.

\section{Adsorption properties examination}

In this research, the solid-phase extraction (SPE) method was used for examination of the adsorption properties of modified SBA sorbents.

The exact amount of material $(300 \mathrm{mg})$ was crumbled in a mortar and placed in an empty extraction cartridge (column) with PTFE frits (6 mL, J.T. Baker).

The following substances were used as adsorbates: camphor, DBE-4, DBE-5, HEMA, DA, CQ, phenol, 4-MP, EDMAB, and TEGDMA. All listed compounds were dissolved in an artificial saliva solution (SAGF) prepared as was described in the literature (Gal et al. 2011). 
The SPE procedure for examination of the adsorption consists of the following steps. The examined sorptive material in extraction cartridge was conditioned with $10 \mathrm{~mL}$ of methanol. After conditioning, $3 \mathrm{~mL}$ of organic compounds' solution $\left(0.1 \mathrm{mg} \mathrm{mL}^{-1}\right.$ or $\left.0.5 \mathrm{mg} \mathrm{mL}^{-1}\right)$ was applied to the sorbent for the adsorption of the analytes. The sample matrix after the adsorption step was collected and in separate stages of research, it was applied to the extraction cartridge with $\mathrm{C} 18$ for transferring the remaining non-adsorbed compounds to the organic solution $(3 \mathrm{~mL}$ of sample matrix was applied on the $\mathrm{C} 18$ layer and flushed with $1 \mathrm{~mL}$ of methanol). The experiments were repeated three times. During the SPE experiments, each portion of the organic solvent after all the stages was collected and examined by gas chromatography (GC).

Table 1 Retention times and correlation coefficients for analyzed compounds
Fig. 1 FTIR spectra of nonmodified material and modified by MTAB or CTAB (a); spectral region $3500-2550 \mathrm{~cm}^{-1}$ (b); spectral region $1600-1400 \mathrm{~cm}^{-1}$ (c); spectral region 1400 $600 \mathrm{~cm}^{-1}$ (d)

\section{GC analysis}

For GC analysis, the Perkin Elmer Clarus 580 chromatograph was used with automatic injector and FID detector. The chromatograph was equipped with capillary column Zebron ZB-WAX $(30 \mathrm{~m} \times 0.25 \mathrm{~mm} \times 0.25 \mu \mathrm{m})$ produced by Phenomenex. Analysis was carried out using helium as the carrier gas at a flow rate of $2.0 \mathrm{ml} / \mathrm{min}$. Injection volume was $1.0 \mu \mathrm{L}$. The injector and detector temperatures were $250{ }^{\circ} \mathrm{C}$. The initial column temperature was $80{ }^{\circ} \mathrm{C}$ (hold for $2 \mathrm{~min}$ ), ramped at $20^{\circ} \mathrm{C} / \mathrm{min}$ to $250{ }^{\circ} \mathrm{C}$ and held constant for $2 \mathrm{~min}$. Total time of analysis was $12.5 \mathrm{~min}$. The amount of compounds in the analyzed solution was read out with the calibration curve method. The correlation coefficients and retention times of examined substances are presented in Table 1.

\section{Results and discussion}

\begin{tabular}{llc}
\hline & $R^{2}$ & $R_{\mathrm{t}}(\min )$ \\
\hline Camphor & 0.998205 & 5523 \\
DBE-4 & 0.997984 & 5977 \\
DBE-5 & 0.997746 & 6644 \\
HEMA & 0.998095 & 7185 \\
DA & 0.997714 & 7332 \\
CQ & 0.998288 & 8246 \\
Phenol & 0.998509 & 8378 \\
4-MP & 0.997712 & 10,235 \\
EDMAB & 0.999561 & 10,906 \\
TEGDMA & 0.997695 & 11,376 \\
\hline
\end{tabular}

The FTIR spectra obtained for modified materials are presented in Figs. 1 and 2. These spectra are presented for selected wavelength ranges. The peaks at 3400 and $1640 \mathrm{~cm}^{-1}$ for all samples are assigned to the stretching vibration and bending vibration of the absorbed water and surface hydroxyl groups, respectively (Figs. 1a, 2a). The $\mathrm{C}-\mathrm{H}$ stretching and anti-stretching vibration frequencies of SBA modified with ammonium bromides are observed at 2857 and $2929 \mathrm{~cm}^{-1}$ (Fig. 1b). The peak at $1480 \mathrm{~cm}^{-1}$ for modified samples (Fig. 1c) is attributed to the $\mathrm{C}-\mathrm{H}$ scissoring vibrations, while the singlet at $948 \mathrm{~cm}^{-1}$ is due

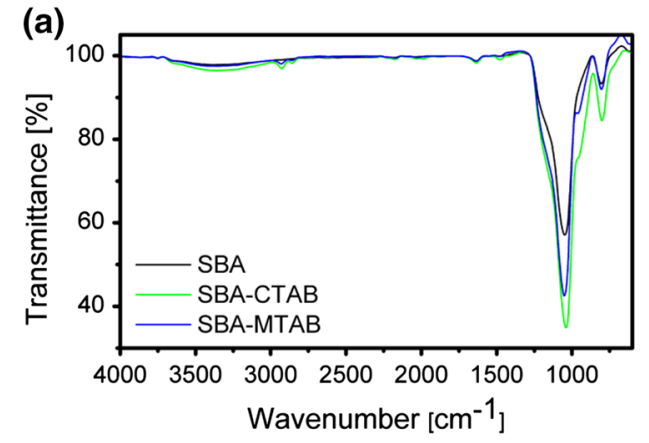

(c)

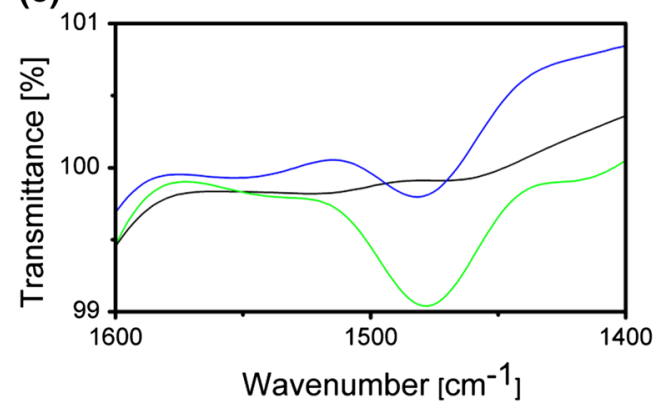

(b)

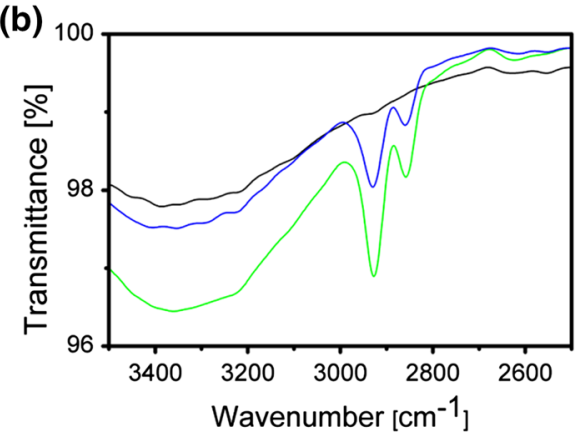

(d)

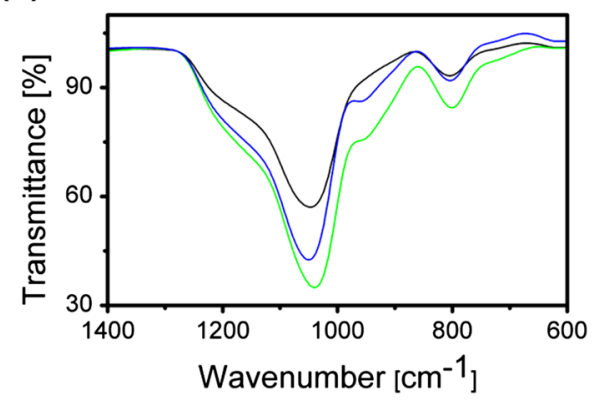


Fig. 2 FTIR spectra of nonmodified SBA material and diazonium-modified materials (a); spectral region 1700 $1550 \mathrm{~cm}^{-1}$ (b); spectral region $1550-1350 \mathrm{~cm}^{-1}(\mathbf{c})$; spectral region $1000-600 \mathrm{~cm}^{-1}$ (d) (a)

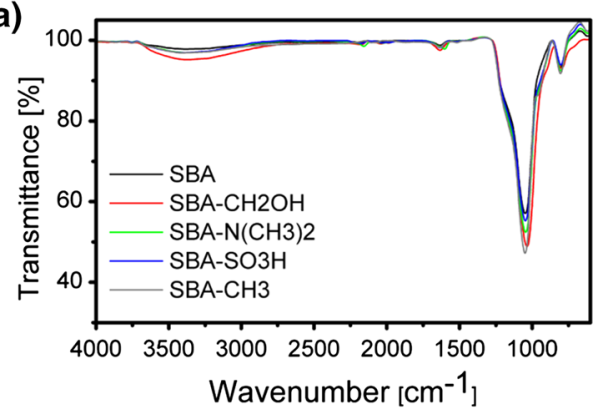

(c)

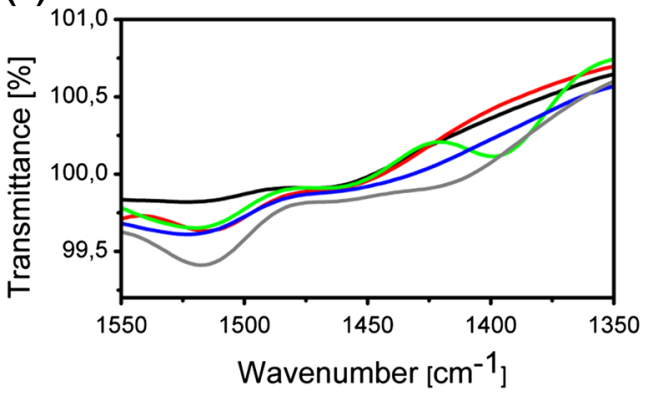

(b)

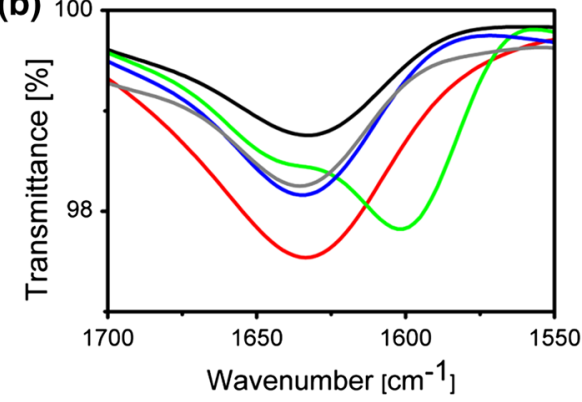

(d)

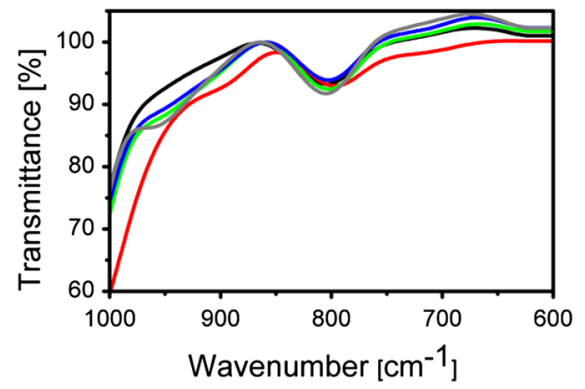

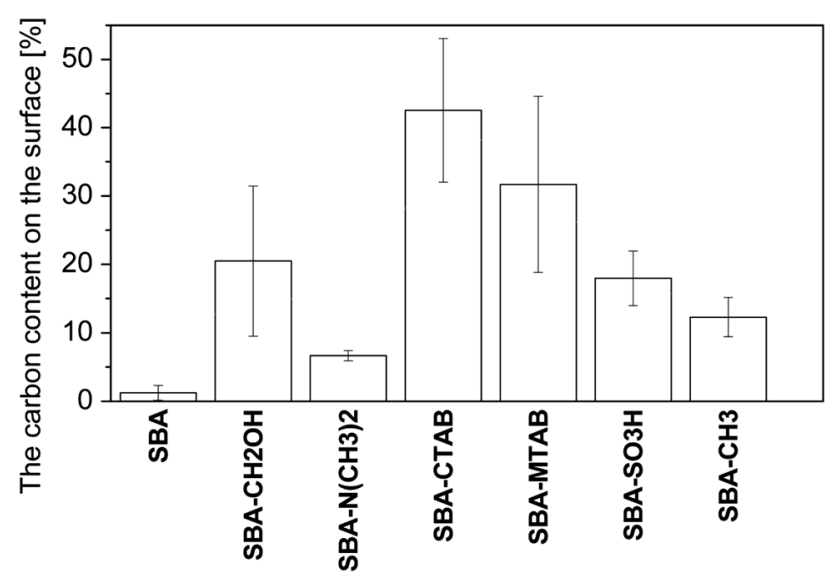

Fig. 3 The carbon content on the surface of unmodified and modified SBA-15 examined using energy-dispersive spectrometry (EDS)

to the C-N stretching bands (Fig. 1d) (Ede et al. 2014; Ding et al. 2017; Guivar et al. 2015). The bands in range $1500-1550 \mathrm{~cm}^{-1}$ are assigned to breathing of aromatic rings and appear in the spectra of all diazonium-modified materials (Fig. 2c). The bands at $1600 \mathrm{~cm}^{-1}$ (Fig. 2b) are assigned to ring breathing, while those at $1400 \mathrm{~cm}^{-1}$ (Fig. 2c) are assigned to $-\mathrm{CH}_{3}$ stretching and appears only in the spectra of SBA-N $\left(\mathrm{CH}_{3}\right)_{2}$ material (Jung et al. 2011).

The effectiveness of modifications is confirmed by the results of the EDS analysis (Fig. 3). The largest increase in the amount of carbon on the surface was observed for samples modified with surfactant. The smallest impact was noted for the sample modified by 4-( $N, N$-dimethyl)benzenediazonium salt; on its surface, the lowest increase in the amount of carbon was noted, but the effectiveness of the modification was clearly demonstrated using the FTIR analysis. In the sample, $\mathrm{SBA}-\mathrm{SO}_{3} \mathrm{H}$ sodium $(0.15 \%)$ was also found. It is related to the ion exchange between the sulfone groups and sodium cations from sodium nitrite during the modification.

The results of subsequent analyses carried out in this work were presented as the difference between the values obtained for unmodified and modified material (2). The following formula was used for the calculation:

Value of modified material - value of unmodified material $=$ value presented

The low temperature nitrogen adsorption method allowed to determine the value of surface area as well as the size and pore volume of the examined sorbents. The surface area of the unmodified material was $857 \mathrm{~m}^{2} / \mathrm{g}$, while the pore volume was $1.2 \mathrm{~cm}^{3} / \mathrm{g}$. The diazonium and surfactant-modified SBA-15 has a smaller surface area and total pore volume than unmodified material (Fig. 4). This dependence in the case of diazonium modification is related to the coverage of surface by the aryl layer. These groups are localized at the entrance of pores and then close or fill them (Toupin and Bélanger 2008). The same relationship was found earlier for carbons modified with diazonium salts ( $\mathrm{Li}$ et al. 2018; Geng et al. 2012; Grondein and Bélanger 2011). The smallest change in both surface and volume was noted for the sample modified using 4-sulfobenzendiazonium salt.

The influence of chain length in the surfactant is also visible. The CTAB-modified material has a smaller specific surface area (Fig. 4). The situation is reversed in the case of pore volume, this is related to facilitating the deposition of MTAB molecules in the pores due to their smaller size. 

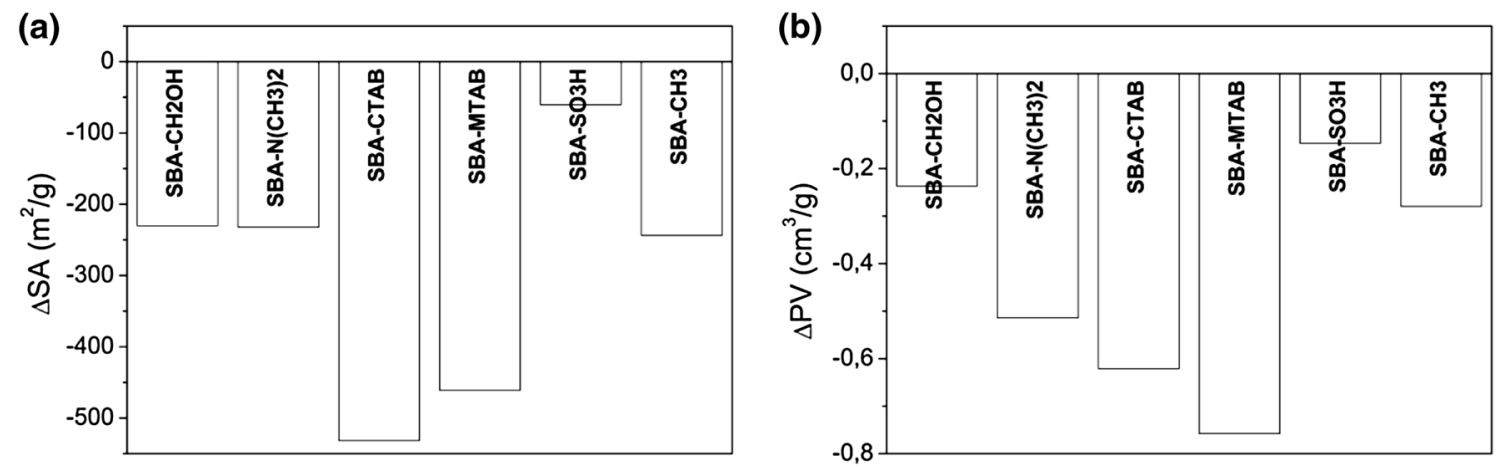

Fig. 4 The difference of surface area $\Delta \mathrm{SA}(\mathbf{a})$ and total pore volume $\Delta \mathrm{PV}(\mathbf{b})$ of the modified materials in relation to the unmodified material

(a)

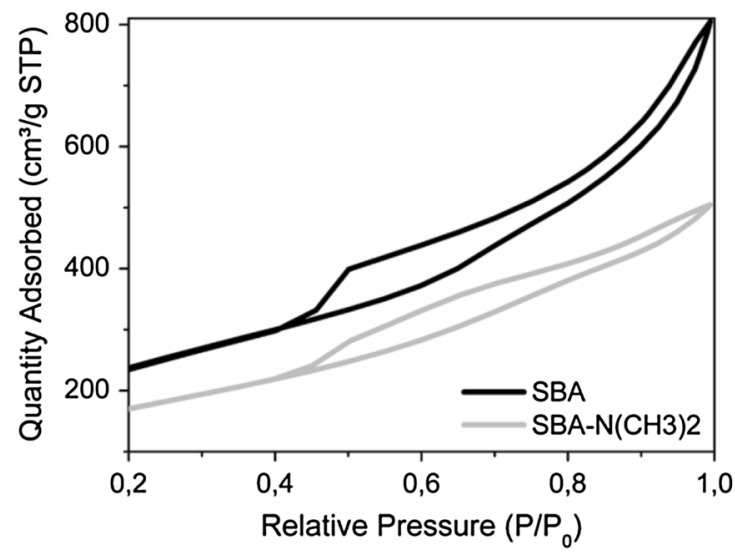

(b)

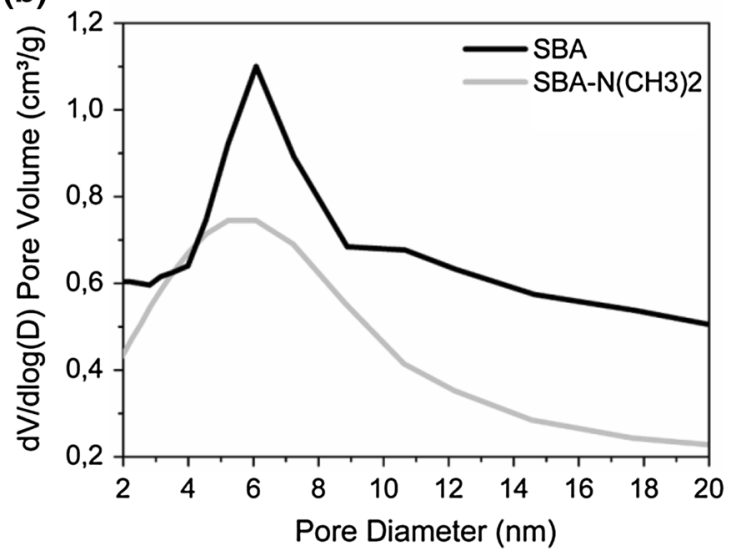

Fig. $5 \mathrm{~N}_{2}$ sorption isotherms (a) and pore size distribution (b) plots of SBA and SBA-N $\left(\mathrm{CH}_{3}\right)_{2}$

An isotherm for the SBA material and the SBA-N $\left(\mathrm{CH}_{3}\right)_{2}$ material is given as an example (Fig. 5a). These samples exhibited a typical type IV adsorption isotherm with a hysteresis loop and peaks in the mesopore range. The same relationship exists in the case of other materials. The material before and after the modification is a mesoporous material (Fig. 5b), but the modification affects the reduction of the pore diameter and consequently, the surface area is lower for modified materials.

The results obtained during the IGC (Fig. 6) analysis indicate a smaller or similar value of the dispersive component of the surface energy of the tested materials in relation to the starting material. The largest decrease of the $\gamma_{s}^{d}$ value was noted for SBA- $\mathrm{CH}_{2} \mathrm{OH}$ and SBA-N $\left(\mathrm{CH}_{3}\right)_{2}$ materials, while the smallest was for $\mathrm{SBA}-\mathrm{CH}_{3}$. Thus, the activity of the SBA- $\mathrm{CH}_{2} \mathrm{OH}$ and SBA-N $\left(\mathrm{CH}_{3}\right)_{2}$ materials' surface expressed by its ability to dispersive interactions is lower than for unmodified material. It might be due to lower surface area. There was a slight increase of surface energy in the case of material SBA- $\mathrm{SO}_{3} \mathrm{H}$. This may be due to the low impact of the modification on the change of the specific surface area of the tested material.

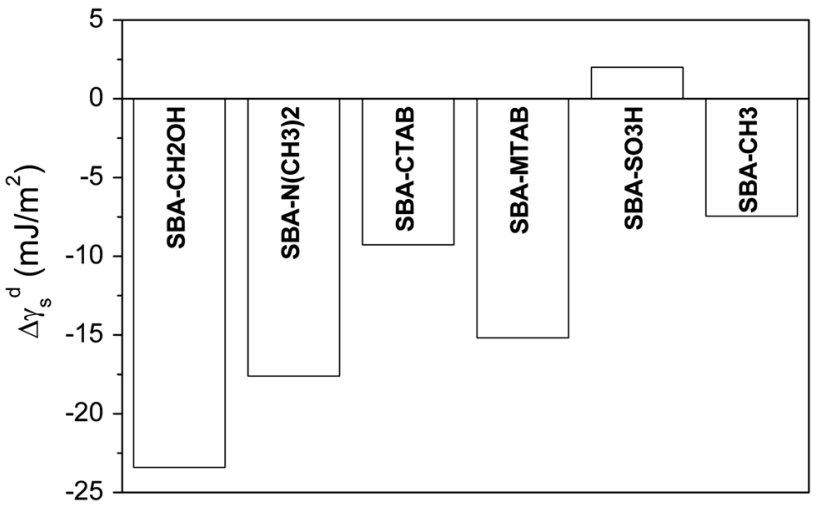

Fig. 6 The difference of the dispersive component of the surface energy $\Delta \gamma_{s}^{d}$ of the modified materials in relation to the unmodified material

The influence of modification of SBA materials on the sorption of individual compounds with respect to unmodified material is presented in the Fig. 7. 

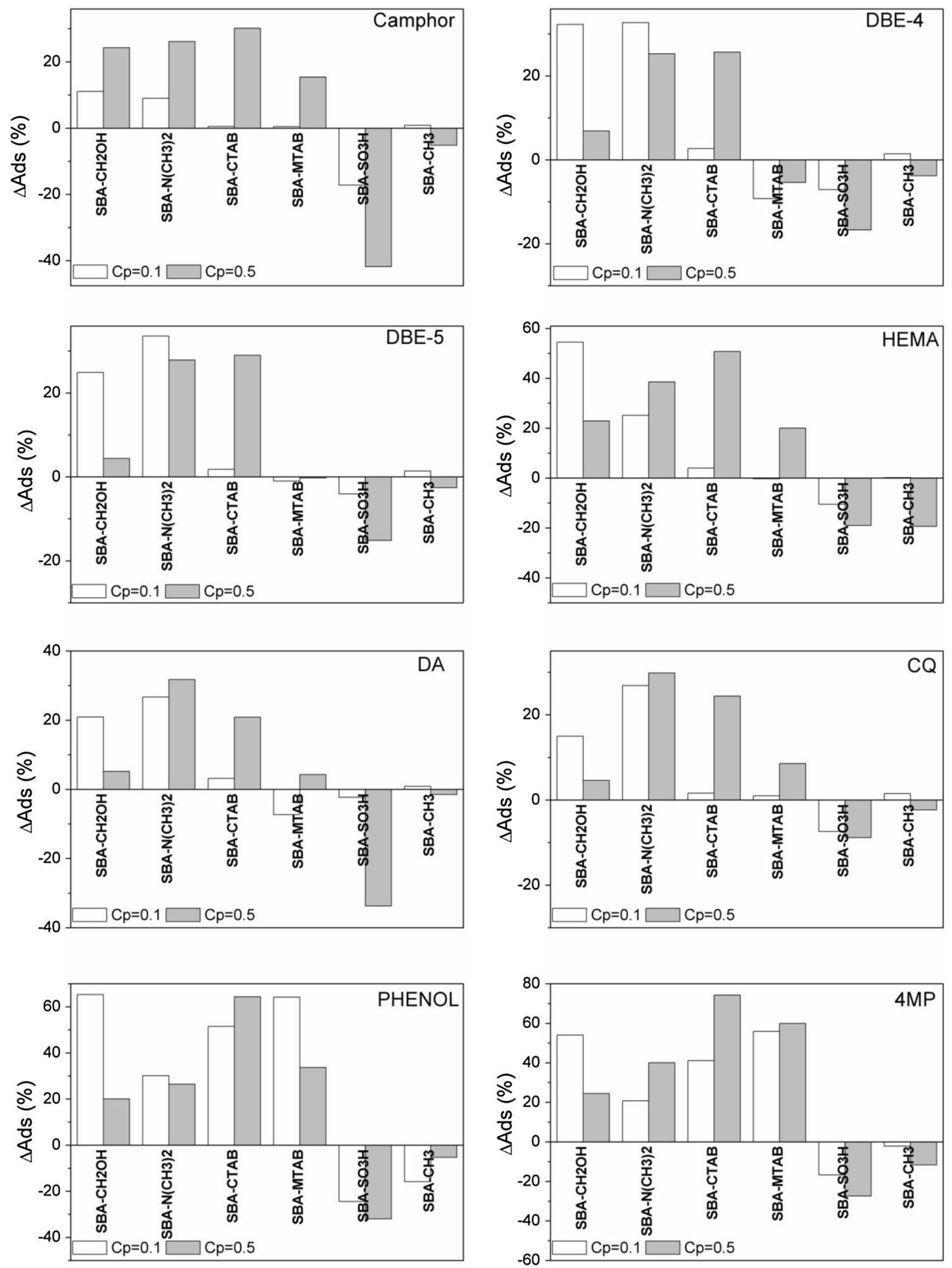

Fig. 7 The difference of adsorption properties $\Delta$ Ads of modified materials in relation to unmodified material for 0.1 and $0.5 \mathrm{mg} \mathrm{mL}^{-1}$ concentration of compounds 
The experiments were repeated at least three times for two concentrations of used adsorbates ( 0.1 and $0.5 \mathrm{mg} \mathrm{mL}^{-1}$ ) and the presented data are referenced to results obtained for non-modified SBA material. Modification of SBA with 4-sulfobenzenediazonium and 4-methylbenzenediazonium salts led to the deterioration of the adsorption properties of modified sorbent. However, despite the smaller surface area, total pore volume and surface energy, the first four materials have better adsorptive properties compared to those of unmodified material. Camphor is better retained on $\mathrm{SBA}-\mathrm{CH}_{2} \mathrm{OH}$ due to the formation of hydrogen bonds between the hydroxyl groups on the surface of the sorbent and the carbonyl groups present in the camphor molecule. In the case of $\mathrm{SBA}-\mathrm{N}\left(\mathrm{CH}_{3}\right)_{2}$, this effect results from strong interactions between the dimethylamine group and the carbonyl group (Kwon et al. 2012). For both materials, these interactions were found for compounds that have carbonyl and ester moieties in their structure (DBE-4, DBE-5, HEMA, DA, CQ). For low concentrations, the adsorption of HEMA is higher for the material SBA- $\mathrm{CH}_{2} \mathrm{OH}$ due to the presence of the hydroxyl group and steric hindrance at the ester group. In the case of phenol, the hydrogen interactions between the hydroxyl groups are stronger than between dimethylamine groups. The same relationships are visible for both concentrations. These results demonstrate the influence of specific interactions on improving the adsorptive properties of materials.

The lack of the influence of modification with surfactants (SBA-CTAB, SBA-MTAB) on the adsorption magnitude of the first six compounds from low concentration solutions is evident. However, such an effect of the modification is visible for the other two adsorbates. This is related to the interactions between hydrophobic benzene ring of phenol or 4-methoxyphenol and the hydrophobic carbon chain according to the mechanism proposed by Xie et al. (2012). A greater effect is visible at higher concentration, and probably results from the hydrophobic interaction of methyl or phenol groups (from compounds) with carbon chains (from surfactant layer) (Chang et al. 2010). A higher impact of the modification using the cetyltrimethylammonium bromide on the adsorption properties was observed, which is related to the length of the carbon chain.

Materials modified with 4-sulfobenzendiazonium salt and 4-methylbenzenediazonium salt deteriorated their adsorption properties. This is due to the fact that the absence of strong chemical interaction between the analytes and organic layer on the surface of the sorbents. The modification affects the reduction of the specific surface area, without forming active sites, leading to the decrease of the adsorption properties. Therefore, even in the case of phenol and methoxyphenol for which the organic layer is similar to the adsorbing molecule, the results are worse after modification.
Both types of modified materials adsorbed the EDMAB and TEGDMA to the same extent as the unmodified sorbent material.

\section{Conclusions}

It was possible to show the applicability of the series of new materials based on Al-SBA-15 modification. The starting material was modified using diazonium salts and surfactants, while the effectiveness of material modification was proved by the results of Fourier transform infrared spectroscopy and energy-dispersive spectrometry. The materials after modification have smaller surface area and pore volume. This change results from coating the surface with an aryl layer and closing the pores. Dispersive component of the free surface energy is also lower for modified materials. Despite the decrease in such important material properties, the adsorptive properties deteriorated for only two modifications (aryl group with sulfone and methyl groups). For other modifications with the use of diazonium salts, the effectiveness of sorption increased and depended on the presence of moieties (phenyl, hydroxyl, ester, methoxy, carbonyl) in the adsorbed compound. The influence of modification with the surfactant in the case of sorption from low concentrations is visible only for phenol and methoxyphenol. The greater effect is visible for a higher concentration, and is connected with the hydrophobic interaction of methyl or phenyl groups in adsorbed compounds with carbon chains of the surfactant layer. The effect of modification with the use of diazonium salts and surfactants on the sorptive properties of materials is evident. In the case of modification by diazonium salt, one can obtain model materials with the properties required for the sorption of the given group of chemicals in an easy way (changing the substitute in the aromatic amine).

Acknowledgements This paper was produced with the financial support from the Polish National Science Centre (Grant no. UMO2015/17/B/ST8/02388). Authors would like to thank Dr. Beata Strzemiecka for helping in IGC experiments' performance and interpretation.

Open Access This article is distributed under the terms of the Creative Commons Attribution 4.0 International License (http://creativeco mmons.org/licenses/by/4.0/), which permits unrestricted use, distribution, and reproduction in any medium, provided you give appropriate credit to the original author(s) and the source, provide a link to the Creative Commons license, and indicate if changes were made.

\section{References}

Chang JH, Lee J, Jeong Y, Lee JH, Kim IJ, Park SE (2010) Hydrophobic partitioning approach to efficient protein separation with magnetic nanoparticles. Anal Biochem 405:135-137 
Cui Y, Hu ZJ, Yang JX, Gao HW (2012) Novel phenyl-iminodiacetic acid grafted multiwalled carbon nanotubes for solid phase extraction of iron, copper and lead ions from aqueous medium. Microchim Acta 176:359-366

Davidson CL, Feilzer AJ (1997) Polymerization shrinkage and polymerization shrinkage stress in polymer-based restoratives. J Dent 25:435-440

Ding S, Xiong X, Liu X, Shi Y, Jianga Q, Hu J (2017) Synthesis and characterization of single-crystalline $\mathrm{Bi}_{2} \mathrm{O}_{2} \mathrm{SiO}_{3}$ nanosheets with exposed 001 facets. Catal Sci Technol 7:3791-3801

Ede SR, Nithiyanantham U, Kundu S (2014) Enhanced catalytic and SERS activities of CTAB stabilized interconnected osmium nanoclusters. Phys Chem Chem Phys 16:22723-22734

Gal JY, Fovet Y, Adib-Yadzi M (2011) About a synthetic saliva for in vitro studiem. Talanta 53:1103-1115

Geng L, Yu G, Wang Y, Zhu Y (2012) Ph-SO ${ }_{3} \mathrm{H}$-modified mesoporous carbon as an efficient catalyst for the esterification of oleic acid. Appl Catal A-Gen 427-428:137-144

Geurtsen W, Lehmann F, Spahl W, Leyhausen G (1998) Cytotoxicity of 35 dental resin composite monomers/additives in permanent $3 \mathrm{~T} 3$ and three human primary fibroblast cultures. J Biomed Mater Res 41:474-480

Gioka C, Bourauel C, Hiskia A, Kletsas D, Eliades T, Eliades G (2005) Light-cured or chemically cured orthodontic adhesive resins? A selection based on the degree of cure, monomer leaching and cytotoxicity. Am J Orthod Dentofacial Orthop 127:413-419

Gomez-Cazalilla M, Merida-Robles JM, Gurbani A, Rodriguez-Castellon E, Jimenez-Lopez A (2007) Characterization and acidic properties of Al-SBA-15 materials prepared by post-synthesis alumination of a low-cost ordered mesoporous silica. J Solid State Chem 180:1130-1140

Grieken R, Escola JM, Moreno J, Rodríguez R (2009) Direct synthesis of mesoporous M-SBA-15 (M= Al, Fe, B, Cr) and application to 1-hexene oligomerization. Chem Eng J 155:442-450

Grondein A, Bélanger D (2011) Chemical modification of carbon powders with aminophenyl and aryl-aliphatic amine groups by reduction of in situ generated diazonium cations: applicability of the grafted powder towards $\mathrm{CO}_{2}$ capture. Fuel 90:2684-2693

Guivar JAR, Sanches EA, Magon CJ, Fernandes EGR (2015) Preparation and characterization of cetyltrimethylammonium bromide (CTAB)-stabilized $\mathrm{Fe} 3 \mathrm{O} 4$ nanoparticles for electrochemistry detection of citric acid. J Electroanal Chem 755:158-166

Hashimoto Y, Nakamura M (2000) Estrogenic activity of dental materials and bisphenol-a related chemicals in vitro. Dent Mater J 19(3):245-262

Heimburger D, Gam-Derouich S, Decorse P, Mangeney C, Pinson J (2016) Reversible trapping of functional molecules at interfaces using diazonium salts chemistry. Langmuir 32:9714-9721

Jung U, Kuhn S, Cornelissen U, Tuczek F, Strunskus T, Zaporojtchenko V, Kubitschke J, Herges R, Magnussen O (2011) Azobenzene-containing triazatriangulenium adlayers on $\mathrm{Au}(111)$ : structural and spectroscopic characterization. Langmuir 27:5899-5908

Koppula SV, Fellman JH, Storrs FJ (1995) Screening allergens for acrylate dermatitis associated with artificial nails. Am J Contact Dermatitis 6:78-85

Kumaran GM, Garg S, Soni K, Kumar M, Gupta JK, Sharma LD, Rao KSR, Dhar GM (2008) Synthesis and characterization of acidic properties of Al-SBA-15 materials with varying $\mathrm{Si} / \mathrm{Al}$ ratios. Micropor Mesopor Mat 114:103-109

Kwon TY, Bagheri R, Kim YK, Kim KH, Burrow MF (2012) Cure mechanisms in materials for use in esthetic dentistry. J Investig Clin Dent 3(1):3-16

Li Z, Burt T, Bowman RS (2000) Sorption of ionizable organic solutes by surfactant-modified zeolite. Environ Sci Technol 34:3756-3760

Li X, Forouzandeh F, Kakanat AJ, Feng F, Banham DWH, Ye S, Kwok DY, Birss V (2018) Surface characteristics of microporous and mesoporous carbons functionalized with pentafluorophenyl groups. ACS Appl Mater Interfaces 10(2):2130-2142

Mahouche-Chergui S, Gam-Derouich S, Mangeney C, Chehimi MM (2011) Aryl diazonium salts: a new class of coupling agents for bonding polymers, biomacromolecules and nanoparticles to surfaces. Chem Soc Rev 40:4143-4166

Manojlovic D, Radisic M, Vasiljevic T, Zivkovic S, Lausevic M, Mileti V (2011) Monomer elution from nanohybrid and ormocerbased composites cured with different light sources. Dent Mater 27(4):371-378

McCabe J (1998) Resin-modified glass-ionomers. Biomaterials 19:521-527

Michelsen VB, Kopperud HB, Lygre GB, Bjorkman L, Jensen E, Kleven IS, Svahn J, Lygre H (2012) Detection and quantification of monomers in unstimulated whole saliva after treatment with resin-based composite fillings in vivo. Eur J Oral Sci 120:89-95

Rogalewicz R, Batko K, Voelkel A (2006) Identification of organic extractables from commercial resin-modified glass-ionomers using HPLC-MS. J Environ Monit 8:750-758

Ruyter IR, Oysaed H (1982) Conversion in dentine base polymers. J Biomed Mater Res 16:741-754

Sandomierski M, Strzemiecka B, Chehimi MM, Voelkel A (2016) Reactive diazonium-modified silica fillers for high-performance polymers. Langmuir 32:11646-11654

Sandomierski M, Buchwald T, Strzemiecka B, Voelkel A (2018a) Modification of Ti6Al4 V surface by diazonium compounds. Spectrochim Acta A 191:27-35

Sandomierski M, Strzemiecka B, Grams J, Chehimi MM, Voelkel A (2018b) Diazonium-modified zeolite fillers. Effect of diazonium substituent position on the filler surface modification and the mechanical properties of phenolic/zeolite composites. Int J Adhes Adhes 85:157-164

Schultz J, Lavielle L, Martin C (1987) Proprietes de surface des fibres de carbone dèterminèes par chromatographie gaze use inverse. $\mathrm{J}$ Chimie Phys 84:231-237

Sitko R, Zawisza B, Malicka E (2012) Modification of carbon nanotubes for preconcentration, separation and determination of tracemetal ions. Trac-Trend Anal Chem 37:22-31

Swift EJ, Pawlus MA, Vargas MA, Fortin D (1995) Depth of cure of resin-modified glass ionomers. Dent Mater 11:196-200

Szczodrowski K, Prélot B, Lantenois S, Douillard JM, Zajac J (2009) Effect of heteroatom doping on surface acidity and hydrophilicity of $\mathrm{Al}, \mathrm{Ti}, \mathrm{Zr}$-doped mesoporous SBA-15. Micropor Mesopor Mat 124:84-93

Toupin M, Bélanger D (2008) Spontaneous functionalization of carbon black by reaction with 4-nitrophenyldiazonium cations. Langmuir 24(5):1910-1917

Voelkel A (2012) Physicochemical measurements by inverse gas chromatography. In: Poole CF (ed) gas chromatography. Elsevier, Amsterdam

Wahab MF, Pohl CA, Lucy CA (2011) Ion chromatography on carbon clad zirconia modified by diazonium chemistry and functionalized latex particles. Analyst 136:3113-3120

Wanga J, Liu QA (2008) Simple method to directly synthesize AlSBA-15 mesoporous materials with different Al contents. Solid State Commun 148:529-533

Wilder AD, Swift EJ, May KN, Thompson JY, McDougal RA (2000) Effect of Finishing technique on the microleakage and surface texture of resin-modified glass ionomer restorative materials. J Dent 28:367-373

Xie J, Meng W, Wu D, Zhang Z, Kong H (2012) Removal of organic pollutants by surfactant modified zeolite: comparison between ionizable phenolic compounds and non-ionizable organic compounds. J Hazard Mater 231-232:57-63 
Xie Q, Xie J, Wang Z, Wu D, Zhang Z, Kong H (2013) Adsorption of organic pollutants by surfactant modified zeolite as controlled by surfactant chain length. Micropor Mesopor Mat 179:144-150

Zeng S, Blanchard J, Breysse M, Shi Y, Shu X, Nie H, Li D (2005) Post-synthesis alumination of SBA-15 in aqueous solution: a versatile tool for the preparation of acidic Al-SBA-15 supports. Micropor Mesopor Mat 85:297-304

Publisher's Note Springer Nature remains neutral with regard to jurisdictional claims in published maps and institutional affiliations. 